\title{
ON THE STABILITY OF THE NYSTRÖM METHOD FOR THE MUSKHELISHVILI EQUATION ON CONTOURS WITH CORNERS
}

\author{
VICTOR D. DIDENKO* AND JOHAN HELSING ${ }^{\dagger}$
}

\begin{abstract}
The stability of the Nyström method for the Muskhelishvili equation on piecewise smooth simple contours $\Gamma$ is studied. It is shown that in the space $L^{2}$ the method is stable if and only if certain operators $A_{\tau_{j}}$ from an algebra of Toeplitz operators are invertible. The operators $A_{\tau_{j}}$ depend on the parameters of the equation considered, on the opening angles $\theta_{j}$ of the corner points $\tau_{j} \in \Gamma$ and on parameters of the approximation method mentioned. Numerical experiments show that there are opening angles where the operators $A_{\tau_{j}}$ are non-invertible. Therefore, for contours with such corners the method under consideration is not stable. Otherwise, the method is always stable. Numerical examples show an excellent convergence of the method.
\end{abstract}

Key words. Muskhelishvili equation, Nyström method, stability

AMS subject classifications. 65R20, 45L05

1. Introduction. Let $\Gamma$ be a simple closed curve in the complex plane $\mathbb{C}$. The Muskhelishvili integral equation

$$
A_{\Gamma, k} \varphi(t) \equiv-k \overline{\varphi(t)}-\frac{k}{2 \pi i} \int_{\Gamma} \overline{\varphi(\tau)} d \log \frac{\bar{\tau}-\bar{t}}{\tau-t}-\frac{1}{2 \pi i} \int_{\Gamma} \varphi(\tau) d \frac{\bar{\tau}-\bar{t}}{\tau-t}=f_{0}(t) t \in \Gamma
$$

plays an important role in the study of various problems in elasticity, hydrodynamics and in other fields of applied mathematics. Thus in planar elastic problems for solid bodies, the trace of the stress tensor on the boundary $\Gamma$ can be expressed directly via the derivative of the function $\varphi$. Other quantities of physical interest, such as displacements and the full stress tensor of the material, can be also extracted from the solution of the equation (1.1), [20, Sections 32 and 98].

The right-hand side $f_{0}$ of the equation (1.1) has a special form - viz.

$$
f_{0}(t)=-\frac{1}{2} \overline{f(t)}+\frac{1}{2 \pi i} \int_{\Gamma} \frac{\overline{f(\tau)} d \tau}{\tau-t}
$$

where $f$ is a given function, and the bar denotes the complex conjugation. The real number $k$ is fixed and can take the value one or it is defined by the Poisson constant $0<\nu<1 / 2$. In the following, we use the notations

$$
k_{1}=1, \quad k_{2}=-(3-4 \nu), \quad k_{3}=-\frac{3-\nu}{1+\nu} .
$$

The equation (1.1) with the coefficient $k=k_{2}$ or $k=k_{3}$ is usually connected to planar elastic problems for solids with prescribed displacements on the boundary $\Gamma$ $[18,19,20,21]$. On the other hand, this equation with coefficient $k=1$ is related to various boundary value problems for biharmonic equation in plane domains, cf. $[8,20,21]$ and references therein. It is remarkable that properties of the operator

\footnotetext{
${ }^{*}$ Faculty of Science, Universiti Brunei Darussalam, Bandar Seri Begawan, BE1410 Brunei (diviol@gmail.com), supported by the Universiti Brunei Darussalam under Grants $\mathrm{UBD} / \mathrm{GSR} / \mathrm{S} \& \mathrm{~T} / 19$ and UBD/PNC2/2/RG/1(159).

†Numerical Analysis, Centre for Mathematical Sciences, Lund University, Box 118, SE-221 00 LUND, Sweden (helsing@maths.1th.se), supported by the Swedish Research Council under Grant 621-2011-5516.
} 
$A_{\Gamma, k}$ allows one to develop convenient, stable and very accurate numerical procedures. There is an extensive literature dealing with various approximation methods for the Muskhelishvili equation. However, for a long time only approximation methods based on Fourier series expansions have been used. This leads to unnecessary restrictions on contour $\Gamma$, viz. it must be a circle or a sufficiently smooth curve. For smooth $\Gamma$ one can make use of conformal mappings and transfer the original Muskhelishvili equation onto the circle [1]. However, such an approach requires an essential additional computational cost. On the other hand, approximation methods using non-trigonometric polynomial bases can be employed to the equation on non-circular contours directly, [13]. The smoothness of $\Gamma$ is also important in other issues related to the stability of the method under consideration. Thus it ensures the compactness of the integral operators from (1.1), so the sequences of approximating operators converge uniformly to the corresponding integral operators cf. $[16,22]$ and the stability of the approximation method depends only on the invertibility of the operator under consideration.

It is notable that very often the application of an approximation method to the Muskhelishvili equation is justified only by certain amount of examples without rigorous analysis of its stability. A systematic study of such problems have been started only recently. Thus in the space $L^{2}(\Gamma)$ the stability of spline Galerkin, spline collocation, and qualocation methods on simple smooth curves has been established in [10]. For such contours, the methods mentioned are always stable. The situation becomes more involved if $\Gamma$ possesses corner points. It turns out now that stability depends on certain operators associated with the corner points of $\Gamma$ and on certain specific parameters of the corresponding approximation method. Nevertheless, for the Galerkin method using piecewise constant splines, necessary and sufficient stability conditions have been obtained [7]. Moreover, in [7] an approximation method based on the rectangular quadrature rule has also been studied. The last two methods mentioned have both advantages and drawbacks. Thus the rectangular rule method can be easily implemented but its convergence is relatively slow. On the other hand, Galerkin methods have a better convergence rate but their implementation requires additional efforts. In particular, one has to approximate the integral terms in the equations involved and also the integrals representing the corresponding scalar products. As a result, it changes the structure of the approximating operators and the stability conditions, cf. [2].

In the present paper, we deal with an approximation method which is based on the composite Gauss-Legendre quadrature formula

$$
\int_{0}^{1} u(s) d s \approx \sum_{l=0}^{n-1} \sum_{p=0}^{d-1} w_{p} u\left(s_{l_{p}}\right) / n .
$$

The points $s_{l p}$ are defined by

$$
s_{l p}:=\frac{l+\varepsilon_{p}}{n}, \quad l=0,1, \ldots, n-1 ; p=0,1, \ldots, d-1,
$$

where $w_{p}$ and $0<\varepsilon_{0}<\varepsilon_{1}, \ldots<\varepsilon_{d-1}<1$ are, respectively, the weights and the Gauss-Legendre points on the interval $[0,1]$, and $d$ is a fixed positive integer. Such a method combines the merits of the rectangular rule and Galerkin methods. It can be easily implemented and it demonstrates an excellent convergence rate. In the process, special grids refined in neighbourhoods of the corner points are used. We 
establish necessary and sufficient conditions of the stability of the method, and it is worth mentioning that the corresponding operators responsible for the stability of the spline Galerkin method from [7] have a simpler structure. Moreover, it is easily seen that those operators are always Fredholm. On the other hand, the operators $A_{\tau_{i}}$ associated with the stability of the present method are much more complicated; their Fredholmness is not as apparent and it will be studied elsewhere. Let us also recall that the Nyström method for another celebrated integral equation of elasticity-viz. for the Sherman-Lauricella equation, has been studied in [4]. Note the Muskhelishvili equation contains an additional parameter $k$ which has to be taken into account while studying Fredholm properties of the initial operator $A_{\Gamma, k}$. Moreover, this situation also requires us to use different invertibility correcting operators $T_{\Gamma, k}$ for different values of $k$, cf. (2.5). Let us also emphasize that the stability of this method depends on the invertibility of certain operators associated with the equation considered, with the parameters of the method and with the opening angles of the corner points of $\Gamma$. As a result, the conditions of the stability of the Nyström method for the Muskhelishvili equation differ from those for the Sherman-Lauricella equation. Nevertheless, for both the Muskhelishvili and Sherman-Lauricella equation the operators responsible for the stability belong to a famous Toeplitz algebra which makes it possible to study their Fredholm properties. However, the structure of these operators is rather complicated and there is no analytical method to find out whether they are invertible or not. Thus in [4] the corresponding operators for the Sherman-Lauricella equation have been described but no verifiable condition of their invertibility was given. In contrast to that work, here we not only establish the stability conditions but also propose an approximation procedure for their verification. It allows us to find critical angles which cause the instability of the method. For example, in the case of the Muskhelishvili equation with the parameter $k=1$, the method we use has 8 singular angles in the interval $[0.1 \pi, 1.9 \pi]$, whereas for $k=-1.5$ the same interval contains only 6 such critical angles, cf. Figures 4.1 and 4.2 .

Note that in all cases, an algebraic approach to study stability is employed. Thus for the equation under consideration the corresponding approximation method is included into a specific $C^{*}$-algebra of operator sequences such that the stability of the method is equivalent to the invertibility of an element in an associated quotient algebra. Consequently, the invertibility of the related coset is studied and stability conditions are obtained.

2. Spaces and operators. Let $X$ be a Banach space over the field of complex numbers $\mathbb{C}$. We say that an operator $A: X \mapsto X$ is additive if $A\left(x_{1}+x_{2}\right)=A x_{1}+A x_{2}$ for all $x_{1}, x_{2} \in X$. As usual, the set of all additive continuous operators on the space $X$ is denoted by $\mathcal{L}_{a d d}(X)$. This set becomes a real $C^{*}$-algebra, if one provides it with natural operations of addition, multiplication, multiplication by real scalars, and with a special norm [8]. Further, if $X$ is a Banach space and if $m$ is a positive integer, then by $X^{m}$ we will denote the Cartesian product of $m$ copies of the space $X$ provided with the norm

$$
\|x\|_{X^{m}}=\left\|\left(x_{1}, \ldots, x_{m}\right)\right\|_{X^{m}}=\left(\sum_{j=1}^{m}\left\|x_{j}\right\|_{X}^{2}\right)^{1 / 2} .
$$

Assume that $\Gamma$ is a simple closed piecewise smooth positively oriented contour in the complex plane $\mathbb{C}$, and let $\gamma=\gamma(s), s \in \mathbb{R}$ be a 1-periodic parametrization of $\Gamma$. Denote by $\mathcal{M}_{\Gamma}$ the set of all corner points $\tau_{0}, \tau_{1}, \ldots, \tau_{q-1}$ of $\Gamma$, and let $\theta=\theta_{\tau} \in(0,2 \pi)$ denote 


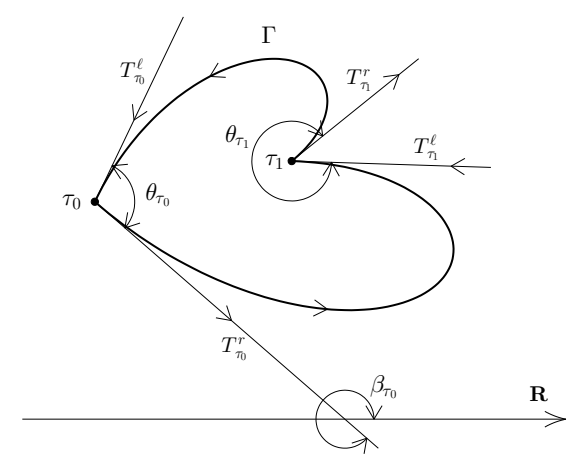

FIG. 2.1. Corner points of $\Gamma: \theta_{\tau}$ is the left angle between the left $T_{\tau}^{l}$ and right $T_{\tau}^{r}$ semi-tangents at the point $\tau \in \Gamma$.

the left angle between the left $T_{\tau}^{l}$ and right $T_{\tau}^{r}$ semi-tangents to $\Gamma$ at the point $\tau$. It is not equal to $\pi$ for any $\tau \in \mathcal{M}_{\Gamma}$. Moreover, let $\beta=\beta_{\tau} \in[0,2 \pi)$ be the angle between the right semi-tangent $T_{\tau}^{r}$ to $\Gamma$ at the point $\tau$ and the real axis $\mathbb{R}$, cf. Fig. 2.1. If $\tau_{j}$ is a corner point, we will write these parameters as $\theta_{j}$ and $\beta_{j}$ rather than $\theta_{\tau_{j}}$ and $\beta_{\tau_{j}}$. Further, there is no loss of generality in assuming that $\tau_{j}=\gamma(j / q)$ for all $j=0,1, \ldots, q-1$. In addition, we will always suppose that the function $\gamma$ is two times continuously differentiable on each interval $(j / q,(j+1) / q)$ and

$$
\left|\gamma^{\prime}\left(\frac{j}{q}+0\right)\right|=\left|\gamma^{\prime}\left(\frac{j}{q}-0\right)\right|, \quad j=0,1, \ldots, q-1 .
$$

Let $C(\Gamma)$ denote the set of all complex-valued functions continuous on $\Gamma$, and let $L^{2}(\Gamma)$ refer to the set of all Lebesgue measurable complex-valued functions $f$ on $\Gamma$ such that

$$
\|f\|_{L^{2}}=\|f\|_{2}:=\left(\int_{\Gamma}|f(\tau)|^{2}|d \tau|\right)^{1 / 2}<\infty
$$

and let $W^{1,2}(\Gamma)$ denote the closure of the set of all functions $f$ with bounded derivatives in the norm

$$
\|\left. f\right|_{W^{1,2}}:=\left(\int_{\Gamma}|f(\tau)|^{2}|d \tau|+\int_{\Gamma}\left|f^{\prime}(\tau)\right|^{2}|d \tau|\right)^{1 / 2} .
$$

In the present paper, the applicability of the original Nyström method to the Muskhelishvili equation in the space $L^{2}(\Gamma)$ is studied. However, in order to establish its stability conditions one first has to investigate the operator $A_{\Gamma, k}$ of (1.1) in both spaces $L^{2}(\Gamma)$ and $W^{1,2}(\Gamma)$.

Let $M$ denote the operator of complex conjugation, i.e.

$$
M \varphi(t):=\overline{\varphi(t)}, \quad t \in \Gamma,
$$

and let $L_{\Gamma}$ and $K_{\Gamma}$ be the integral operators

$$
L_{\Gamma} \varphi(t):=\frac{1}{2 \pi i} \int_{\Gamma} \varphi(\tau) d \ln \left(\frac{\tau-t}{\bar{\tau}-\bar{t}}\right), \quad K_{\Gamma}:=\frac{1}{2 \pi i} \int_{\Gamma} \varphi(\tau) d\left(\frac{\bar{\tau}-\bar{t}}{\tau-t}\right) .
$$


Then the operator $A_{\Gamma, k}$ of (1.1) has the form

$$
A_{\Gamma, k}=-k M+k M L_{\Gamma}-K_{\Gamma}
$$

Consider the smallest closed real $C^{*}$-subalgebra $\mathcal{B}_{2}(\Gamma):=\mathcal{B}_{2}\left(S_{\Gamma}, M, C(\Gamma)\right)$ of the algebra $\mathcal{L}_{a d d}\left(L^{2}(\Gamma)\right)$, which contains the Cauchy singular integral operator $S_{\Gamma}$,

$$
S_{\Gamma} f(t):=\frac{1}{\pi i} \int_{\Gamma} \frac{f(\tau) d \tau}{\tau-t}
$$

the operator of complex conjugation $M$, and the operators of multiplication by functions $f \in C(\Gamma)$. It is known that $L_{\Gamma}, K_{\Gamma} \in \mathcal{B}_{2}(\Gamma)$, [11]. Moreover, if $f \in C(\Gamma)$ then the operator $f S_{\Gamma}-S_{\Gamma} f$ is compact and the set $\mathcal{K}_{a d d}\left(L^{2}(\Gamma)\right)$ of all compact operators is contained in $\mathcal{B}_{2}(\Gamma)[17]$. For the reader's convenience, we also recall some results of $[7,8]$ concerning the operator $A_{\Gamma, k} \in \mathcal{B}_{2}(\Gamma)$ and adapt them to the situation considered here.

Let $\mathbb{R}^{+}$refer to the set of all positive real numbers. Fix $\theta \in(0,2 \pi)$, and consider the operators $\mathcal{N}_{\theta}, \mathcal{M}_{\theta}: L^{2}\left(\mathbb{R}^{+}\right) \rightarrow L^{2}\left(\mathbb{R}^{+}\right)$defined by

$$
\begin{aligned}
& \mathcal{N}_{\theta}(f)(\sigma):=\frac{1}{2}\left(\frac{1}{2 \pi i} \int_{0}^{+\infty} \frac{f(s) d s}{s-\sigma e^{i \theta}}-\frac{1}{2 \pi i} \int_{0}^{+\infty} \frac{f(s) d s}{s-\sigma e^{i(2 \pi-\theta)}}\right) \\
& \mathcal{M}_{\theta} f(\sigma):=\frac{1}{\pi} \int_{0}^{+\infty}\left(\frac{\sigma}{s}\right) \frac{\sin \theta}{\left(1-(\sigma / s) e^{i \theta}\right)^{2}} \frac{f(s)}{s} d s
\end{aligned}
$$

With each corner point $\tau_{j} \in \mathcal{M}_{\Gamma}$ we associate an operator $A_{\theta_{j}}:\left(L^{2}\left(\mathbb{R}^{+}\right)\right)^{4} \rightarrow$ $\left(L^{2}\left(\mathbb{R}^{+}\right)\right)^{4}$ defined by

$$
\begin{aligned}
& A_{\theta_{j}}= \\
& \left(\begin{array}{cccc}
0 & e^{-i 2 \beta_{j}} \mathcal{M}_{\theta_{j}} & -k I & k \mathcal{N}_{\theta_{j}} \\
-e^{-i 2\left(\beta_{j}+\theta_{j}\right)} \mathcal{M}_{2 \pi-\theta_{j}} & 0 & k \mathcal{N}_{\theta_{j}} & -k I \\
-k I & k \mathcal{N}_{\theta_{j}} & 0 & -e^{i 2 \beta_{j}} \mathcal{M}_{2 \pi-\theta_{j}} \\
k \mathcal{N}_{\theta_{j}} & -k I & e^{i 2\left(\beta_{j}+\theta_{j}\right)} \mathcal{M}_{\theta_{j}} & 0
\end{array}\right)
\end{aligned}
$$

where $I$ is the identity operator on the space $L^{2}\left(\mathbb{R}^{+}\right)$.

LEMMA 2.1. If $\Gamma$ is a simple closed piecewise continuous contour, then the Muskhelishvili operator $A_{\Gamma, k}: L^{2}(\Gamma) \rightarrow L^{2}(\Gamma)$ is Fredholm if and only if all operators $A_{\theta_{j}}:\left(L^{2}\left(\mathbb{R}^{+}\right)\right)^{4} \rightarrow\left(L^{2}\left(\mathbb{R}^{+}\right)\right)^{4}$ are invertible.

Note that the entries of the matrix $A_{\theta_{j}}$ are Mellin operators, so the invertibility of such operators can be studied. Thus if one introduces the functions

$$
\mathbf{n}_{\theta}(z)=\frac{\sinh (\pi-\theta) z}{\sinh \pi z}, \quad \mathbf{m}_{\theta}(z)=-e^{-i \theta} \frac{z \sin \theta}{\sinh \pi z} e^{-(\theta-\pi) z}, \quad z=x+i / 2, \quad x \in \mathbb{R}
$$

then the invertibility of the operator $A_{\theta_{j}}$ can be described as follows.

LEMma 2.2. The operator $A_{\theta_{j}}$ is invertible if and only if the determinant of the 
matrix

$$
\begin{aligned}
& \Phi_{\theta_{j}}(z)= \\
& \left(\begin{array}{cccc}
0 & e^{-i 2 \beta_{j}} \mathbf{m}_{\theta_{j}}(z) & -k & k \mathbf{n}_{\theta_{j}}(z) \\
-e^{-i 2\left(\beta_{j}+\theta_{j}\right)} \mathbf{m}_{2 \pi-\theta_{j}}(z) & 0 & k \mathbf{n}_{\theta_{j}}(z) & -k \\
-k & k \mathbf{n}_{\theta_{j}}(z) & 0 & -e^{i 2 \beta_{j}} \mathbf{m}_{2 \pi-\theta_{j}}(z) \\
k \mathbf{n}_{\theta_{j}}(z) & -k & e^{i 2\left(\beta_{j}+\theta_{j}\right)} \mathbf{m}_{\theta_{j}}(z) & 0
\end{array}\right)
\end{aligned}
$$

is bounded away from zero on the line $L:=\{z \in \mathbb{C}: z=x+i / 2, x \in \mathbb{R}\}$.

It turns out that the determinant of the matrix $\Phi_{\theta_{j}}$ can be represented in a convenient form. That allows one to provide a complete solution to the invertibility problem of the operators $A_{\theta_{j}}$.

LEMMA 2.3. For any parameters $\theta_{j} \in(0,2 \pi)$ and $\beta_{j} \in[0,2 \pi)$ the operator $A_{\theta_{j}}:\left(L^{2}\left(\mathbb{R}^{+}\right)\right)^{4} \rightarrow\left(L^{2}\left(\mathbb{R}^{+}\right)\right)^{4}$ is invertible.

Proof. Expanding the determinant $\operatorname{det} \Phi_{\theta_{j}}(z)$ by the first two or by the last two rows, one obtains

$$
\operatorname{det} \Phi_{\theta_{j}}(z)=\frac{\left[z^{2} \sin ^{2}\left(2 \pi-\theta_{j}\right)-k^{2} \sinh ^{2}\left(\left(2 \pi-\theta_{j}\right) z\right)\right]\left[z^{2} \sin ^{2} \theta_{j}-k^{2} \sinh ^{2}\left(\theta_{j} z\right)\right]}{\sinh ^{4}(\pi z)}
$$

Consider now the function

$$
\psi_{\theta_{j}}(z)=z^{2} \sin ^{2} \theta_{j}-k^{2} \sinh ^{2}\left(\theta_{j} z\right) .
$$

Since $|k|>1$ and

$$
\begin{aligned}
\sinh \left(\theta_{j} z\right) & =\sinh \left(\left(x+\frac{i}{2}\right) \theta_{j}\right)=\sinh \left(\theta_{j} x+\frac{\theta_{j}}{2} i\right) \\
& =\sinh \left(\theta_{j} x\right) \cos \left(\frac{\theta_{j}}{2}\right)+\cosh \left(\theta_{j} x\right) \sin \left(\frac{\theta_{j}}{2}\right) i
\end{aligned}
$$

the imaginary part of the function $k \sinh \left(\theta_{j} z\right), z=x+i / 2, x \in \mathbb{R}$ can be estimated as follows

$$
\begin{aligned}
\mid \operatorname{Im}\left(k \sinh \left(\theta_{j} z\right) \mid\right. & =|k|\left|\cosh \left(x \theta_{j}\right)\right|\left|\sin \left(\frac{\theta_{j}}{2}\right)\right| \\
& \geq\left|\sin \left(\frac{\theta_{j}}{2}\right)\right|>\frac{\left|\sin \theta_{j}\right|}{2}=\left|\operatorname{Im}\left(z \sin \theta_{j}\right)\right| .
\end{aligned}
$$

Thus if $\operatorname{Re} \psi_{\theta_{j}}(z)=0$, then $\operatorname{Im} \psi_{\theta_{j}}(z) \neq 0$, so $\psi_{\theta_{j}}$ does not vanish on the line $L$. Therefore, the function $\Phi_{\theta_{j}}$ is bounded away from zero, which implies the invertibility of the operator $A_{\theta_{j}}$ for any $\theta_{j} \in(0,2 \pi)$ and $\beta_{j} \in[0,2 \pi)$. $\square$

For $k \in\left\{k_{1}, k_{2}, k_{3}\right\}$, let $T_{\Gamma, k}$ be the operator defined by

$$
\begin{aligned}
& T_{\Gamma, k} \varphi(t):= \\
& \begin{cases}\frac{1}{2 \pi i} \int_{\Gamma} \frac{\varphi(\tau) d \tau}{\tau}+\frac{1}{t} \frac{1}{2 \pi i} \int_{\Gamma}\left(\frac{\varphi(\tau) d \tau}{\tau^{2}}+\frac{\overline{\varphi(\tau) d \tau}}{\bar{\tau}^{2}}\right) & \text { if } k=k_{1} \\
\frac{1}{2 \pi i} \int_{\Gamma} \frac{\varphi(\tau) d \tau}{\tau} & \text { if } k \in\left\{k_{2}, k_{3}\right\}\end{cases}
\end{aligned}
$$



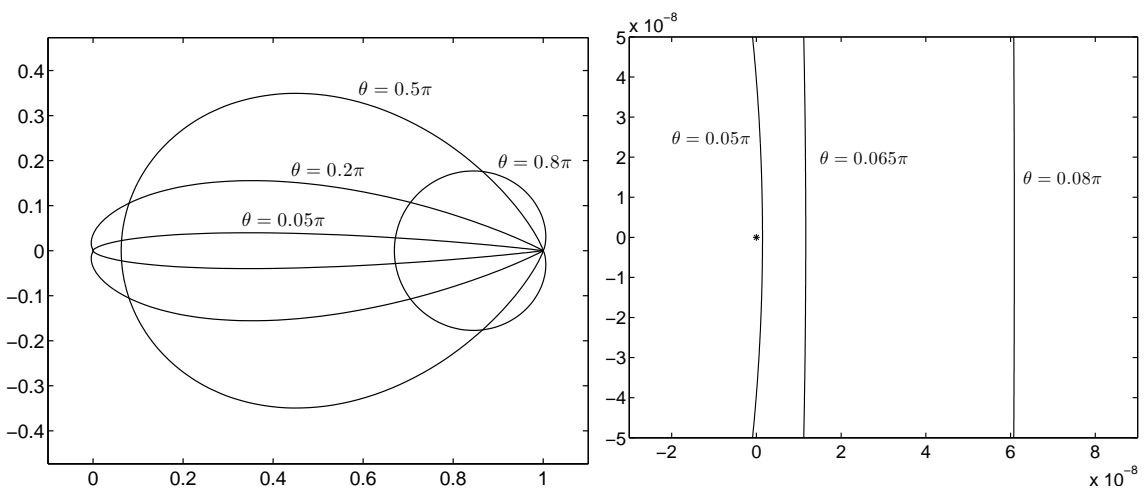

FIG. 2.2. Left: The graph of the contour $\Omega_{\Gamma}$ when $\Gamma$ has corners with various opening angles $\theta$ and $k=1$. The contour is symmetric with respect to $\pi$ in the sense that it is identical for corners with opening angle $\theta$ and $2 \pi-\theta$. Right: Zoom of the graph of $\Omega_{\Gamma}$ in the neighbourhood of the origin for small opening angles $\theta$. The origin is marked by the asterisk. It is remarkable that even if some coils are located extremely close to the origin, neither $\Omega_{\Gamma}$ nor its interior contains the origin, i.e. the operator $B_{\Gamma, 1}$ is Fredholm and ind $B_{\Gamma, 1}=0$.

Now we can formulate the main result needed to construct stable approximation methods for the Muskhelishvili equation (1.1) in the space $L^{2}(\Gamma)$.

THEOREM 2.4. Let $\Gamma$ be a simple closed piecewise continuous contour. Then for any $k \in\left\{k_{1}, k_{2}, k_{3}\right\}$ the operator $B_{\Gamma, k}:=A_{\Gamma, k}+T_{\Gamma, k}: L^{2}(\Gamma) \rightarrow L^{2}(\Gamma)$ is invertible. Moreover, if a function $f$ belongs to the space $W^{1,2}(\Gamma)$ and satisfies the condition

$$
\operatorname{Re} \int_{\Gamma} \overline{f(\tau)} d \tau=0
$$

then the solution of the equation

$$
B_{\Gamma, k} \varphi=f
$$

is a solution of the equation (1.1).

Proof. The contour $\Gamma$ and operator $A_{\Gamma, k}$ define the function

$$
\omega(\tau, z):=\left\{\begin{array}{ll}
\operatorname{det} \Phi_{\theta}(z) & \text { if } \tau \in \mathcal{M}_{\Gamma}, z \in L \\
k^{4} & \text { if } \tau \in \Gamma \backslash \mathcal{M}_{\Gamma}
\end{array} .\right.
$$

According to Lemmas 2.1-2.3 and [7, Theorem 12], the operator $A_{\Gamma, k}: L^{2}(\Gamma) \rightarrow L^{2}(\Gamma)$ is Fredholm. Since any integral operator $T_{\Gamma, k}$ is compact, the operator $B_{\Gamma, k}: L^{2}(\Gamma) \rightarrow$ $L^{2}(\Gamma)$ is also Fredholm and the index

$$
\text { ind }\left.B_{\Gamma, k}\right|_{L^{2}(\Gamma)}=\left.\operatorname{ind} A_{\Gamma, k}\right|_{L^{2}(\Gamma)}=\text { wind } \Omega_{\Gamma},
$$

where wind $\Omega_{\Gamma}$ is the winding number of the curve $\Omega_{\Gamma}:=\{\omega(\tau, z) \in \mathbb{C}: \tau \in \Gamma, z \in L\}$ which represents the image of the function (2.8) when the initial contour $\Gamma$ is run in the counterclockwise direction. Further, standard homotopy arguments ensure that this index is actually equal to zero, cf. Fig. 2.2.

Thus

$$
\text { ind }\left.B_{\Gamma, k}\right|_{L^{2}(\Gamma)}=0
$$


Moreover, it is known [11] that the operator $B_{\Gamma, k}$ considered on the space $W^{1,2}(\Gamma)$ possesses two distinctive properties - viz.

$$
\text { ind }\left.B_{\Gamma, k}\right|_{W^{1,2}(\Gamma)}=0,\left.\quad \operatorname{ker} B_{\Gamma, k}\right|_{W^{1,2}(\Gamma)}=\{0\} .
$$

Since the space $W^{1,2}(\Gamma)$ is dense in $L^{2}(\Gamma)$, the relations (2.10)-(2.11) imply [12] that

$$
\left.\operatorname{ker} B_{\Gamma, k}\right|_{L^{2}(\Gamma)}=\{0\} \text {. }
$$

Applying (2.10) once more, one obtains that the operator $B_{\Gamma, k}$ is invertible on the space $L^{2}(\Gamma)$, as well.

The second statement of Theorem 2.4 follows from the fact that for any solution $\varphi_{0} \in W^{1,2}(\Gamma)$ of the equation $(2.7)$ one has

$$
T_{\Gamma, k} \varphi_{0}=0 .
$$

This completes the proof. $\square$

It is well-known that the invertibility of the operator associated with the equation under consideration is a necessary condition for the applicability of various approximation methods. The original Muskhelishvili equation does not satisfy this requirementthe operator $A_{\Gamma, k}$ is not invertible in the space $L^{2}(\Gamma)$. However, now we have an invertible operator $B_{\Gamma, k}$ with the property that the solution of the equation (2.7) is a solution of the Muskhelishvili equation (1.1). Therefore, an approximate solution of (1.1) can be derived via approximation of the equation (2.7).

3. The Nyström method and its stability.. Let $d$ be a positive integer and let $0<\varepsilon_{0}<\varepsilon_{1}<\ldots<\varepsilon_{d-1}<1$ and $0<\delta_{0}<\delta_{1}<\ldots<\delta_{d-1}<1$ be real numbers. Consider two sets of points on $\Gamma$,

$$
\tau_{l p}=\gamma\left(\frac{l+\varepsilon_{p}}{n}\right), \quad t_{l p}=\gamma\left(\frac{l+\delta_{p}}{n}\right), \quad l=0,1, \ldots, n-1 ; p=0,1, \ldots, d-1 .
$$

If $K: L^{2}(\Gamma) \rightarrow L^{2}(\Gamma)$,

$$
K \varphi(t):=\int_{\Gamma} k(t, \tau) \varphi(\tau) d \tau
$$

is an integral operator with a sufficiently smooth kernel $k$ and if $\varphi$ is a Riemann integrable function, we approximate the integral (3.2) by the quadrature rule (1.3). Thus

$$
\begin{gathered}
\int_{\Gamma} k(t, \tau) \varphi(\tau) d \tau=\int_{0}^{1} k(\gamma(\sigma), \gamma(s)) \varphi(\gamma(s)) \gamma^{\prime}(s) d s \\
\approx K^{(\varepsilon, n)} \varphi(t)=\sum_{l=0}^{n-1} \sum_{p=0}^{d-1} w_{p} k\left(t, \tau_{l p}\right) \varphi\left(\tau_{l p}\right) \tau_{l p}^{\prime} / n
\end{gathered}
$$

where $\tau_{l p}^{\prime}=\gamma^{\prime}\left(\left(l+\varepsilon_{p}\right) / n\right)$. Note that the kernels $k(t, \tau)$ of the integral operators $K_{\Gamma}$ and $L_{\Gamma}$ possess finite limits $\lim _{t \rightarrow \tau} k(t, \tau)$ for any $\tau \notin \mathcal{M}_{\Gamma}[10]$. This allows us to define the expression $k(\tau, \tau)$ by

$$
k(\tau, \tau):=\lim _{t \rightarrow \tau} k(t, \tau), \quad \tau \notin \mathcal{M}_{\Gamma} .
$$


Therefore, if $k$ is the kernel of the operator $K_{\Gamma}$ or $L_{\Gamma}$, then the expression $k\left(\tau_{l p}, \tau_{l p}\right)$ is correctly defined.

Now we can approximate the solution of equation (2.7) at the points $\tau_{l p}, l=$ $0,1, \ldots, n-1, p=0,1, \ldots, d-1$. Set $n=q m, m=1,2, \ldots$ and note that for such $n$, any corner of $\Gamma$ always is the end point of some an interval $(\gamma(j / n), \gamma((j+1) / n))$. We replace all integrals in (2.7) by the corresponding terms derived from the quadrature formula (3.3) and equate the expressions obtained for the left-hand side of (2.7) and the function $f_{0}$ at the points $t_{k r}$, replacing the terms $\varphi\left(t_{l r}\right)$ by $\varphi\left(\tau_{l r}\right)$ everywhere. As the result, approximate values $\widetilde{\varphi}_{n}\left(\tau_{l r}\right)$ of the solution $\varphi$ of the equation (2.7) at the points $\tau_{l r}, l=0,1, \ldots, n-1, r=0,1, \ldots, d-1$ can be derived from the following system of algebraic equations

$$
\begin{aligned}
& -k \overline{\widetilde{\varphi}_{n}\left(\tau_{k r}\right)}-\frac{k}{2 \pi i} \sum_{l=0}^{n-1} \sum_{p=0}^{d-1} w_{p} \overline{\widetilde{\varphi}_{n}\left(\tau_{l p}\right)}\left(\frac{\overline{\tau^{\prime}} l_{p}}{\bar{\tau}_{l p}-\bar{t}_{k r}}-\frac{\tau_{l p}^{\prime}}{\tau_{l p}-t_{k r}}\right) \frac{1}{n} \\
& -\frac{1}{2 \pi i} \sum_{l=0}^{n-1} \sum_{p=0}^{d-1} w_{p} \overline{\widetilde{\varphi}_{n}\left(\tau_{l p}\right)}\left(\frac{\tau_{l p}-t_{k r}}{\left(\bar{\tau}_{l p}-\bar{t}_{k r}\right)^{2}} \frac{\overline{\tau_{l p}}}{n}-\frac{1}{\bar{\tau}_{l p}-\bar{t}_{k r}} \frac{\tau_{l p}^{\prime}}{n}\right) \\
& \quad+\left(T_{\Gamma, k}^{(\varepsilon, n)} \widetilde{\varphi}_{n}\right)\left(t_{k r}\right)=f\left(t_{k r}\right), k=0,1, \ldots, n-1 ; r=0,1, \ldots, d-1 .
\end{aligned}
$$

Let $(d+1) / n<1$, and let $S_{n}^{d}(\Gamma)$ denote the space of the smoothest splines of degree $d$ on $\Gamma$ associated with the parametrization $\gamma: \mathbb{R} \rightarrow \Gamma$, cf. [4] or [8, Sections 5.3 and 5.5] for more details. Having obtained approximate values $\widetilde{\varphi}_{n}$ of the solution $\varphi$ at the points $\tau_{l p}, l=0,1, \ldots, n-1, p=0,1, \ldots, d-1$, one can construct an interpolation spline $\varphi_{n} \in S_{n}^{d}(\Gamma)$, which satisfies the relation

$$
\varphi_{n}\left(\frac{l+\delta_{p}}{n}\right)=\widetilde{\varphi}_{n}\left(\frac{l+\delta_{p}}{n}\right) \text { for all } l=0,1, \ldots, n-1, \quad p=0,1, \ldots, d-1,
$$

and approximates an exact solution of the equation (1.1) on the whole curve $\Gamma$.

Let $Q_{n}^{\delta}: L_{\infty}(\Gamma) \mapsto S_{n}^{d}(\Gamma)$ denote the interpolation projection on the subspace $S_{n}^{d}(\Gamma)$ such that

$$
Q_{n}^{\delta} \varphi\left(t_{l p}\right)=\varphi\left(t_{l p}\right), \quad l=0,1, \ldots, n-1 ; p=0,1, \ldots, d-1
$$

for each function $\varphi$ from the set of the Riemann integrable functions $\mathbf{R}(\Gamma)$. It is known [22] that under some mild assumptions about the set $\left\{\delta_{p}\right\}_{0}^{d-1}$ such projections exist, and the sequence $\left(Q_{n}^{\delta}\right)_{n \in \mathbb{N}}: \mathbf{R}(\Gamma) \mapsto L_{2}(\Gamma)$ strongly converges to the corresponding embedding operator ,

$$
\lim _{n \rightarrow \infty}\left\|Q_{n}^{\delta} f-f\right\|_{L_{2}(\Gamma)}=0, \quad f \in \mathbf{R}(\Gamma) .
$$

Moreover, let $P_{n}^{d}: L_{2}(\Gamma) \mapsto S_{n}^{d}(\Gamma)$ be the orthogonal projection onto the spline space $S_{n}^{d}(\Gamma)$. Then the system of algebraic equations (3.4) is equivalent to the following operator equation

$$
\begin{aligned}
& Q_{n}^{\delta} B_{\Gamma, k}^{(\varepsilon, n)} P_{n}^{d} \varphi_{n} \equiv-k Q_{n}^{\delta} P_{n}^{d} M \varphi_{n}+k Q_{n}^{\delta} M L_{\Gamma}^{(\varepsilon, n)} P_{n}^{d} \varphi_{n} \\
& \quad-Q_{n}^{\delta} M K_{\Gamma}^{(\varepsilon, n)} P_{n}^{d} \varphi_{n}+Q_{n}^{\delta} T_{\Gamma, k}^{(\varepsilon, n)} P_{n}^{d} \varphi_{n}=Q_{n}^{\delta} f, \quad \varphi_{n} \in S_{n}^{d}(\Gamma), \quad n \in \mathbb{N} .
\end{aligned}
$$


As was already mentioned, the aim of this work is to study the applicability of the Nyström method (3.4),(3.7) to the Muskhelishvili equation. The most demanding task in such an investigation is the proof of the stability of the corresponding method. As soon as it is established, it implies the solvability of the systems (3.4) for sufficiently large $n$ and the $L^{2}$-convergence of approximate solutions (3.5) to the exact solution of equation (2.7). Moreover, error estimates can be easily obtained from known results of approximation theory

Definition 3.1. The sequence $\left(Q_{n}^{\delta} B_{\Gamma, k}^{(\varepsilon, n)} P_{n}^{d}\right)$ is called stable if there is an $n_{0} \in \mathbb{N}$ such that for all $n \geq n_{0}$ the operators $Q_{n}^{\delta} B_{\Gamma, k}^{(\varepsilon, n)} P_{n}^{d} P_{n}^{d}: S_{n}^{d}(\Gamma) \mapsto S_{n}^{d}(\Gamma)$ are invertible and the norms $\left\|\left(Q_{n}^{\delta} B_{\Gamma, k}^{(\varepsilon, n)} P_{n}^{d}\right)^{-1} P_{n}^{d}\right\|_{n \geq n_{0}}$ are uniformly bounded.

To study the stability of the sequence $\left(Q_{n}^{\delta} B_{\Gamma, k}^{(\varepsilon, n)} P_{n}^{d}\right)$ we will consider a $C^{*}$-algebra of operator sequences. Let $\mathcal{A}^{\Gamma}$ denote the set of all sequences $\left(A_{n}\right)_{n \in \mathbb{N}}$ of bounded additive operators $A_{n}: S_{n}^{d} \rightarrow S_{n}^{d}$ such that there is an operator $A \in \mathcal{L}_{\text {add }}\left(L^{2}\right)$ with the property

$$
\lim _{n \rightarrow \infty} A_{n} P_{n}^{d} \varphi=A \varphi \text { and } \lim _{n \rightarrow \infty} A_{n}^{*} P_{n}^{d} \varphi=A^{*} \varphi \text { for any } \varphi \in L^{2}(\Gamma) .
$$

In other words, the sequences $\left(A_{n} P_{n}^{d}\right)$ and $\left(A_{n}^{*} P_{n}^{d}\right)$ converge in the strong operator topology to the operators $A$ and $A^{*}$, respectively. In the following, the strong convergence of the sequence $\left(A_{n} P_{n}^{d}\right)$ to an operator $A$ is denoted by

$$
A:=s-\lim _{n \rightarrow \infty} A_{n} P_{n}^{d} .
$$

The set $\mathcal{A}^{\Gamma}$ can be provided with natural componentwise operations of multiplication, addition, involution, multiplication by real scalars, and with the norm

$$
\left\|\left(A_{n}\right)\right\|=\sup \left\|A_{n}\right\|
$$

so it becomes a real $C^{*}$-algebra.

For the following we need a general result about the stability of sequences from the algebra $\mathcal{A}^{\Gamma}$. Let $\mathcal{K}_{a d d}\left(L^{2}(\Gamma)\right) \subset \mathcal{L}_{a d d}\left(L^{2}(\Gamma)\right)$ denote the set of all additive compact operators on the space $L^{2}(\Gamma)$. Let $\mathcal{J}^{\Gamma}$ denote the subset of $\mathcal{A}^{\Gamma}$ that consists of all sequences $\left(J_{n}\right)$ of the form

$$
J_{n}=P_{n}^{d} K P_{n}^{d}+G_{n}
$$

where $K \in \mathcal{K}_{a d d}\left(L^{2}(\Gamma)\right)$ and $\lim _{n \rightarrow \infty}\left\|G_{n}\right\|=0$. $\mathcal{A}^{\Gamma}$.

Lemma $3.2([8])$. The set $\mathcal{J}^{\Gamma}$ is a closed two-sided ideal of the real $C^{*}$-algebra

Let us also recall a general result about the stability of sequences from the alge$\operatorname{bra} \mathcal{A}^{\Gamma}$.

Theorem 3.3 (cf. [8, Proposition 1.6.4]). A sequence $\left(A_{n}\right) \in \mathcal{A}^{\Gamma}$ is stable if and only if the operator $A=s-\lim _{n \rightarrow \infty} A_{n}$ is invertible in $\mathcal{L}_{\text {add }}\left(L^{2}(\Gamma)\right)$ and the coset $\left(A_{n}\right)+\mathcal{J}^{\Gamma}$ is invertible in the quotient algebra $\mathcal{A}^{\Gamma} / \mathcal{J}^{\Gamma}$.

This theorem can be used to study the stability of the Nyström method (3.4),(3.7).

Let $\mathbf{u}=\mathbf{u}_{\theta}$ denote one of the functions (2.3) defined on the line $L:=\{z \in \mathbb{C}: z=$ $x+i / 2, x \in \mathbb{R}\}$. On the space $l_{2}$ of sequences $\left(\xi_{k}\right)$ of complex numbers $\xi_{k}, k=0,1, \ldots$,

$$
l_{2}:=\left\{\left(\xi_{k}\right)_{k=0}^{\infty}: \sum_{k=0}^{\infty}\left|\xi_{k}\right|^{2}<\infty\right\}
$$


the function $\mathbf{u}$ defines bounded linear operators $A_{r, p}^{\delta_{r}, \varepsilon_{p}}, r, p=0,1, \ldots, d-1$, with the matrix representation

$$
A_{r, p}^{\delta_{r}, \varepsilon_{p}}=A_{r, p}^{\delta_{r}, \varepsilon_{p}}(\mathbf{u})=\left(\mathbf{u}\left(\frac{k+\delta_{r}}{l+\varepsilon_{P}}\right) \frac{1}{l+\varepsilon_{p}}\right)_{k, l=0}^{\infty},
$$

where $\varepsilon_{r}, \delta_{r}$ are the parameters in the Nyström method (3.4), (3.7). As the next step, one has to construct an operator $B^{\delta, \varepsilon}(\mathbf{u})$,

$$
B^{\delta, \varepsilon}=B^{\delta, \varepsilon}(\mathbf{u}):=\left(w_{p} A_{r p}^{\delta_{r}, \varepsilon_{p}}(\mathbf{u})\right)_{r, p=0}^{d-1} .
$$

which acts on the space $l_{2}^{d}$. We also need an additional operator $\bar{M}$ defined on the space $l_{2}$ by

$$
\bar{M}\left(\left(\xi_{k}\right)_{k=0}^{\infty}\right):=\left(\bar{\xi}_{k}\right)_{k=0}^{\infty},
$$

and redefined correspondingly on the Cartesian products of $l_{2}$ spaces.

Now we can establish the conditions of applicability of the method considered.

THEOREM 3.4. Let $\Gamma$ be a simple closed piecewise smooth contour. The Nyström method (3.4), (3.7) is stable if and only if the operators $A_{\tau_{j}}$,

$$
\begin{aligned}
A_{\tau_{j}}:=\left(\begin{array}{cc}
0 & e^{i 2 \beta_{j}} B^{\delta, \varepsilon}\left(\boldsymbol{m}_{2 \pi-\theta_{j}}\right) \\
-e^{i 2\left(\beta_{j}+\theta_{j}\right)} B^{1-\delta, 1-\varepsilon}\left(\boldsymbol{m}_{\theta_{j}}\right) & 0
\end{array}\right) \\
+\left(\begin{array}{cc}
-k I & k B^{\delta, \varepsilon}\left(\boldsymbol{n}_{\theta_{j}}\right) \\
k B^{1-\delta, 1-\varepsilon}\left(\boldsymbol{n}_{\theta_{j}}\right) & -k I
\end{array}\right) \bar{M},
\end{aligned}
$$

$\tau_{j} \in \mathcal{M}_{\Gamma}$ are invertible for all $\tau_{j} \in \mathcal{M}_{\Gamma}$. Moreover, if the right-hand side $f \in W^{1,2}(\Gamma)$ and satisfies the condition (2.6), then the approximate solutions $\varphi_{n} \in S_{n}^{d}$ constructed according to the rule (3.5) converge to an exact solution $\varphi$ of the Muskhelishvili equation (1.1) in $L^{2}$-norm and

$$
\left\|\varphi_{n}-\varphi\right\|_{L^{2}} \leq \frac{c}{n}
$$

where $c$ is a constant independent of $n$.

Proof. Relation (3.6) and the strong convergence of the sequence $\left(P_{n}^{d}\right)$ to the identity operator $I[22]$ imply that the sequence $\left(Q_{n}^{\delta} B_{\Gamma, k}^{(\varepsilon, n)} P_{n}^{d}\right)$ belongs to the real $C^{*}$-algebra $\mathcal{A}^{\Gamma}$ and

$$
\lim _{n \rightarrow \infty} Q_{n}^{\delta} B_{\Gamma, k}^{(\varepsilon, n)} P_{n}^{d}=B_{\Gamma, k} .
$$

Therefore, Theorem 3.3 can be used to study the stability of the Nyström method (3.4), (3.7). Moreover, since the sequence $\left(Q_{n}^{\delta} B_{\Gamma, k}^{(\varepsilon, n)} P_{n}^{d}\right)$ converges to the operator $B_{\Gamma, k}$ and since this operator is invertible by Theorem 2.4, one only has to establish the invertibility of the $\operatorname{coset}\left(Q_{n}^{\delta} B_{\Gamma, k}^{(\varepsilon, n)} P_{n}^{d}\right)+\mathcal{J}^{\Gamma}$ in the real $C^{*}$-algebra $\mathcal{A}^{\Gamma} / \mathcal{J}^{\Gamma}$. Let us recall that there are very developed tools to investigate the invertibility in Banach and $C^{*}$-algebras. Nevertheless, the algebra $\mathcal{A}^{\Gamma}$ is too large in order to treat this problem directly. In connection with this, let us consider a smaller algebra containing our sequence - viz. let $\mathcal{B}^{\Gamma}$ be the smallest real $C^{*}$-subalgebra of $\mathcal{A}^{\Gamma}$ which contains all sequences of the form $\left(Q_{n}^{\delta} B P_{n}^{d}\right)$ with $B \in \mathcal{B}_{2}(\Gamma)$ and the sequences $\left(G_{n}\right), G_{n}$ : 
$S_{n}^{d}(\Gamma) \mapsto S_{n}^{d}(\Gamma)$ with $\lim _{n \rightarrow \infty}\left\|G_{n}\right\| \rightarrow 0$. Note that the ideal $\mathcal{J}^{\Gamma} \subset \mathcal{B}^{\Gamma}$, so $\mathcal{B}^{\Gamma} / \mathcal{J}^{\Gamma}$ is a $C^{*}$-subalgebra of the quotient $C^{*}$-algebra $\mathcal{A}^{\Gamma} / \mathcal{J}^{\Gamma}$. Moreover, it follows from [6, Corollary 10] and [8, Corollary 1.4.10] that the real subalgebra $\mathcal{B}^{\Gamma} / \mathcal{J}^{\Gamma}$ is inverse closed in $\mathcal{A}^{\Gamma} / \mathcal{J}^{\Gamma}$. Thus the coset $\left(Q_{n}^{\delta} B_{\Gamma, k}^{(\varepsilon, n)} P_{n}^{d}\right)+\mathcal{J}^{\Gamma}$ is invertible in $\mathcal{A}^{\Gamma} / \mathcal{J}^{\Gamma}$ if and only if it is invertible in $\mathcal{B}^{\Gamma} / \mathcal{J}^{\Gamma}$. Further, to study invertibility in $\mathcal{B}^{\Gamma} / \mathcal{J}^{\Gamma}$ one can use a localizing principle. Recall that localizing principles are very efficient, and they have been successfully used in approximation methods for singular integral equations with conjugation [5], Mellin integral equations with conjugation [9], the Muskhelishvili and Sherman-Lauricella equations on piecewise smooth curves [4, 7]. For the convenience of the reader, we reformulate the Allan's local principle for real $C^{*}$-algebras here.

Consider a real $C^{*}$-algebra $\mathcal{C}$ and a strictly real closed $C^{*}$-subalgebra $\mathcal{U}$ of the centre of $\mathcal{C}$. Let $\mathfrak{M}$ be the space of maximal ideals of $\mathcal{U}$. If $\tau \in \mathfrak{M}$, then we denote by $\mathcal{I}_{\tau}$ the smallest closed two-sided ideal of $\mathcal{C}$ containing the ideal $\tau$.

Theorem 3.5 (cf. [8, Theorem 1.9.5]). Let $\mathcal{C}$ and $\mathcal{U}$ be as above. An element $c \in \mathcal{C}$ is invertible in $\mathcal{C}$ if and only if for any $\tau \in \mathfrak{M}$ the coset $c+\mathcal{I}_{\tau}$ is invertible in the quotient algebra $\mathcal{C} / \mathcal{I}_{\tau}$.

Let $C_{\mathbb{R}}(\Gamma)$ denote the set of all continuous real-valued functions on $\Gamma$. Consider the smallest closed $C^{*}$-subalgebra $\mathcal{U}^{\Gamma}$ of the algebra $\mathcal{B}^{\Gamma}$ which contains all sequences $\left(F_{n}\right)$ of the form $F_{n}=Q_{n}^{\delta} f P_{n}^{d}+G_{n}$ where $f \in C_{\mathbb{R}}(\Gamma)$ and $\left(G_{n}\right) \in \mathcal{J}^{\Gamma}$. Since for any $f_{1}, f_{2} \in C_{\mathbb{R}}(\Gamma)$ one has

$$
Q_{n}^{\delta} f_{1} P_{n}^{d} Q_{n}^{\delta} f_{2} P_{n}^{d}=Q_{n}^{\delta} f_{1} Q_{n}^{\delta} f_{2} P_{n}^{d}=Q_{n}^{\delta} f_{1} f_{2} P_{n}^{d}=Q_{n}^{\delta} f_{2} P_{n}^{d} Q_{n}^{\delta} f_{1} P_{n}^{d},
$$

and $f S_{\Gamma}-S_{\Gamma} f \in \mathcal{K}_{a d d}\left(L^{2}(\Gamma)\right)[17]$, the algebra $\mathcal{U}^{\Gamma} / \mathcal{J}^{\Gamma}$ is a central subalgebra of $\mathcal{B}^{\Gamma} / \mathcal{J}^{\Gamma}$. Moreover, it is isometrically isomorphic to the real function algebra $C_{\mathbb{R}}(\Gamma)$, so it is strictly real closed $C^{*}$-subalgebra of $\mathcal{B}^{\Gamma} / \mathcal{J}^{\Gamma}$, the space of maximal ideals $\mathfrak{M}$ of which is homeomorphic to $\Gamma$ and the maximal ideal associated with a point $\tau \in \Gamma$ is

$$
\left\{\left(Q_{n}^{\delta} f_{\tau} P_{n}^{d}\right)+\mathcal{J}^{\Gamma}: f_{\tau}(\tau)=0, \quad f_{\tau} \in C_{\mathbb{R}}(\Gamma)\right\} .
$$

Now Theorem 3.5 can be employed to establish the conditions of the invertibility of the coset $\left(Q_{n}^{\delta} B_{\Gamma, k}^{(\varepsilon, n)} P_{n}^{d}\right)+\mathcal{J}^{\Gamma}$ in $\mathcal{B}^{\Gamma} / \mathcal{J}^{\Gamma}$. More precisely, let $\mathcal{I}_{\tau}$ be the smallest closed two-sided ideal of $\mathcal{B}^{\Gamma} / \mathcal{J}^{\Gamma}$ generated by the ideal (3.11). By Theorem 3.5, the $\operatorname{coset}\left(Q_{n}^{\delta} B_{\Gamma, k}^{(\varepsilon, n)} P_{n}^{d}\right)+\mathcal{J}^{\Gamma}$ in $\mathcal{B}^{\Gamma} / \mathcal{J}^{\Gamma}$ is invertible in $\mathcal{B}^{\Gamma} / \mathcal{J}^{\Gamma}$ if and only if the cosets $\left(\left(Q_{n}^{\delta} B_{\Gamma, k}^{(\varepsilon, n)} P_{n}^{d}\right)+\mathcal{J}^{\Gamma}\right)+\mathcal{I}_{\tau}$ are invertible in $\left(\mathcal{B}^{\Gamma} / \mathcal{J}^{\Gamma}\right) / \mathcal{I}_{\tau}$ for all $\tau \in \Gamma$.

Consider first the case $\tau \notin \mathcal{M}_{\Gamma}$. Let $U_{\tau}$ be a neighbourhood of $\tau$, and let $\chi_{U_{\tau}}$ denote the characteristic function of $U_{\tau}$. If $U_{\tau} \cap \mathcal{M}_{\Gamma}=\emptyset$, then the operators $\chi_{U_{\tau}} L_{\Gamma} \chi_{U_{\tau}}, \chi_{U_{\tau}} K_{\Gamma} \chi_{U_{\tau}}$ and $\chi_{U_{\tau}} T_{\Gamma, k} \chi_{U_{\tau}}$ are compact, hence

$$
\left(\left(Q_{n}^{\delta} B_{\Gamma, k}^{(\varepsilon, n)} P_{n}^{d}\right)+\mathcal{J}^{\Gamma}\right)+\mathcal{I}_{\tau}=\left(\left(-M \widetilde{P}_{n}^{d}\right)+\mathcal{J}^{\Gamma}\right)+\mathcal{I}_{\tau} .
$$

The coset in the right-hand side of (3.12) is obviously invertible since so is the operator $-M$. Therefore, if $\tau \notin \mathcal{M}_{\Gamma}$, the coset $\left(\left(Q_{n}^{\delta} B_{\Gamma, k}^{(\varepsilon, n)} P_{n}^{d}\right)+\mathcal{J}^{\Gamma}\right)+\mathcal{I}_{\tau}$ is invertible in $\left(\mathcal{B}^{\Gamma} / \mathcal{J}^{\Gamma}\right) / \mathcal{I}_{\tau}$.

If $\tau \in \mathcal{M}_{\Gamma}$, the situation is more involved and requires a more detailed study. We start with a simple observation that since operators $T_{\Gamma, k}$ in (2.5) are compact, the sequences $\left(Q_{n}^{\delta} B_{\Gamma, k}^{(\varepsilon, n)} P_{n}^{d}\right)$ and $\left(Q_{n}^{\delta} A_{\Gamma, k}^{(\varepsilon, n)} P_{n}^{d}\right)$ belong to the same quotient class of the algebra $\mathcal{B}^{\Gamma} / \mathcal{J}^{\Gamma}$. Thus instead of $\left(Q_{n}^{\delta} B_{\Gamma, k}^{(\varepsilon, n)} P_{n}^{d}\right)+\mathcal{J}^{\Gamma}$ one can use the notation $\left(Q_{n}^{\delta} A_{\Gamma, k}^{(\varepsilon, n)} P_{n}^{d}\right)+\mathcal{J}^{\Gamma}$. 


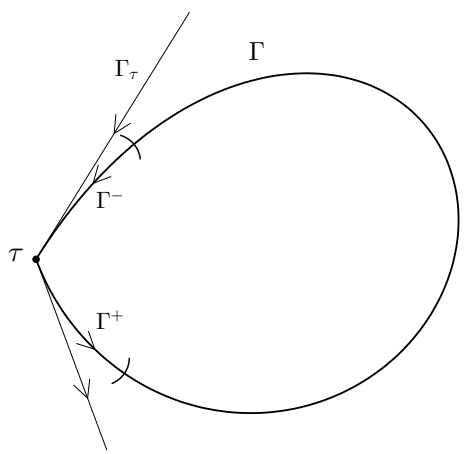

Fig. 3.1. Curves $\Gamma$ and $\Gamma_{\tau}$.

With any $\tau \in \mathcal{M}_{\Gamma}$ we associate a specific curve $\Gamma_{\tau}$, viz. the union of two rays

$$
\Gamma_{\tau}=e^{i\left(\beta_{\tau}+\theta_{\tau}\right)} \mathbb{R} \cup e^{i \beta_{\tau}} \mathbb{R},
$$

where $\theta_{\tau} \in(0,2 \pi)$ is the angle between the right and the left semi-tangents to $\Gamma$ at the point $\tau$, and $\beta_{\tau}$ is the angle between the right semi-tangent to $\Gamma$ at the point $\tau$ and the real axis $\mathbb{R}$.

Now one can consider the Muskhelishvili equation on $\Gamma_{\tau}$ and construct the sequence of Nyström methods

$$
\widetilde{Q}_{n}^{\delta} A_{\Gamma_{\tau}, k}^{(\varepsilon, p)} \widetilde{P}_{n}^{\delta} \widetilde{\varphi}_{n}=f_{n}, \quad n \in \mathbb{N},
$$

where $A_{\Gamma_{\tau}, k}^{(\varepsilon, p)}$ are the approximations of the Muskhelishvili operator $A_{\Gamma_{\tau}, k}$ based on quadratures similar to the quadrature rule (1.3)-(1.4), cf. [4, formula (3.5)], whereas $\widetilde{Q}_{n}^{\delta}$ and $\widetilde{P}_{n}^{\delta}$ are interpolation and orthogonal projections on the corresponding spline spaces on the curve $\Gamma_{\tau}$.

Let $\mathcal{B}_{2}\left(\Gamma_{\tau}\right):=\mathcal{B}_{2}\left(S_{\Gamma_{\tau}}, M, C\left(\Gamma_{\tau}\right)\right)$ be the smallest closed $C^{*}$-subalgebra of the algebra $\mathcal{L}_{\text {add }}\left(L^{2}\left(\Gamma_{\tau}\right)\right)$ constructed analogously to the algebra $\mathcal{B}_{2}(\Gamma)$. Moreover, let $\mathcal{A}^{\Gamma_{\tau}}, \mathcal{B}^{\Gamma_{\tau}}, \mathcal{J}^{\Gamma_{\tau}}$ be the corresponding $C^{*}$-algebras of sequences and the ideals associated with the curve $\Gamma_{\tau}$.

On the contour $\Gamma$ we choose a neighbourhood $U_{\tau}$ of the corner point $\tau$ which does not contain other points from $\mathcal{M}_{\Gamma}$. The neighbourhood $U_{\tau}$ consists of two simple smooth arcs $\Gamma^{-}$and $\Gamma^{+}$, cf. Figure 3.1. Let $t \in \Gamma^{-} \cup \Gamma^{+} \mapsto \Gamma_{\tau}$ and let $l(\tau, t)$ be the length of the corresponding arc connecting these two points. Consider now the mapping $\psi: \Gamma^{-} \mapsto e^{i\left(\beta_{\tau}+\theta_{\tau}\right)} \mathbb{R}$ and $\psi: \Gamma^{+} \mapsto e^{i \beta_{\tau}} \mathbb{R}$ which sends a point $t \in \Gamma^{-} \cup \Gamma^{+}$into $\psi(t) \in \Gamma_{\tau}$ such that $l(\tau, t)=l(\tau, \psi(t))$. Let $V_{\tau}:=\psi\left(U_{\tau}\right)$. The mapping $\psi: U_{\tau} \mapsto V_{\tau}$ is obviously invertible. Moreover, the operator $\psi^{-1} S_{\Gamma_{\tau}} \psi-$ $S_{\Gamma}$ is compact in the space $L^{2}\left(U_{\tau}\right)$ [17], whereas $\psi^{-1} f \psi$ is again an operator of multiplication by a continuous function for any $f \in C_{\mathbb{R}}\left(U_{\tau}\right)$. It implies that the local algebras $\left(\mathcal{B}^{U_{\tau}} / \mathcal{J}^{U_{\tau}}\right) / I_{\tau}$ and $\left(\mathcal{B}^{V_{\tau}} / \mathcal{J}^{V_{\tau}}\right) / \widetilde{I}_{\tau}$ are topologically isomorphic and the cosets $\left(\left(Q_{n}^{\delta} A_{\Gamma, k}^{(\varepsilon, n)} P_{n}^{d}\right)+\mathcal{J}^{\Gamma}\right)+\mathcal{I}_{\tau}$ and $\left(\left(\widetilde{Q}_{n}^{\delta} A_{\Gamma_{\tau}, k}^{(\varepsilon, n)} \widetilde{P}_{n}^{d}\right)+\mathcal{J}^{\Gamma_{\tau}}\right)+\widetilde{\mathcal{I}}_{\tau}$ are simultaneously invertible or not. However, the last coset is invertible if and only if the sequence $\left(\widetilde{Q}_{n}^{\delta} A_{\Gamma_{\tau}, k}^{(\varepsilon, p)} \widetilde{P}_{n}^{\delta}\right)$ is stable. Setting now $\tau:=\tau_{j}$ and following the considerations of Lemma 3.3 and Corollary 3.4 of [4] one obtains that the sequence $\left(\widetilde{Q}_{n}^{\delta} A_{\Gamma_{\tau_{j}}, k}^{(\varepsilon, p)} \widetilde{P}_{n}^{\delta}\right)$ is stable if and only if the operator $A_{\tau_{j}}$ defined by (3.8) is invertible. This completes the proof of the stability part. 
On the other hand, the stability implies the convergence of the approximate solutions $\varphi_{n}$ to the exact solution $\varphi$ of the equation (2.7) in $L^{2}$-norm. By the second part of Theorem 2.4, this function $\varphi$ is simultaneously a solution of the Muskhelishvili equation (1.1). It remains to obtain estimate (3.9). It follows from [22, p.44], and known results on spline approximation [23].
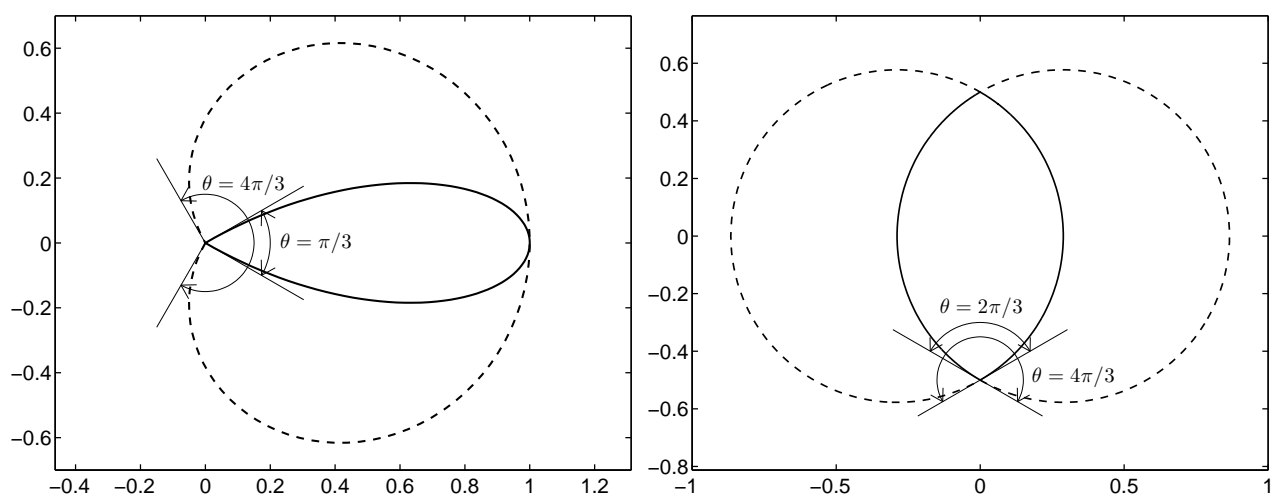

FIG. 3.2. The two contour-types used. Both contour-types are parameterized with an angle $\theta$. Left: a one-corner contour with $\theta=\pi / 3$ (solid line) and $\theta=4 \pi / 3$ (dashed line). Right: a two-corner contour with $\theta=2 \pi / 3$ (solid line) and $\theta=4 \pi / 3$ (dashed line).

3.1. Numerical examples. We end this section with a few numerical examples that illustrate the convergence of the Nyström method (3.4) for (2.7). For this, and for further use in Section 4, we introduce two contours, one of which possesses only one corner point with an opening angle $\theta$ whereas another has two corner points, both with the same opening angle $\theta$, see Figure 3.2.

The one-corner contour in the left image of Figure 3.2 has the parameterization

$$
\gamma(s)=\sin (\pi s) \exp (\mathrm{i} \theta(s-0.5)), \quad s \in[0,1] .
$$

The two-corner contour in the right image of Figure 3.2 is the union of two circular arcs with parameterizations

$$
\begin{array}{ll}
\gamma_{1}(s)=-0.5 \cot (\theta / 2)+0.5 / \sin (\theta / 2) \exp (\mathrm{i} \theta(s-0.5)), & s \in[0,1], \\
\gamma_{2}(s)=0.5 \cot (\theta / 2)-0.5 / \sin (\theta / 2) \exp (\mathrm{i} \theta(s-0.5)), & s \in[0,1],
\end{array}
$$

The special case of $\theta=2 \pi / 3$ makes the two-corner contour assume the shape of a Vesica Piscis.

We consider the above described Nyström method with $d=16$ and with both sets of points $\left\{\epsilon_{p}\right\}$ and $\left\{\delta_{p}\right\}$ of (3.1) coinciding with the zeros of the Legendre polynomial $P_{16}(x)$ on the canonical interval $x \in[-1,1]$, scaled and shifted to the interval $x \in[0,1]$. This corresponds to composite 16-point Gauss-Legendre quadrature.

Figure 3.3 shows approximate solutions $\phi$ to (2.7) obtained for the two-corner contour (3.15)-(3.16) and for various choices of the right-hand side $f$, the parameter $k$, and opening angle $\theta$. One can see that if the oscillation of the argument of the right-hand side $f$ is high, then opening angles close to $2 \pi$ give solutions that are simple closed curves while smaller opening angles give solutions that are increasingly coiled, although the number of coils stabilizes after some point. On the other hand, if the 

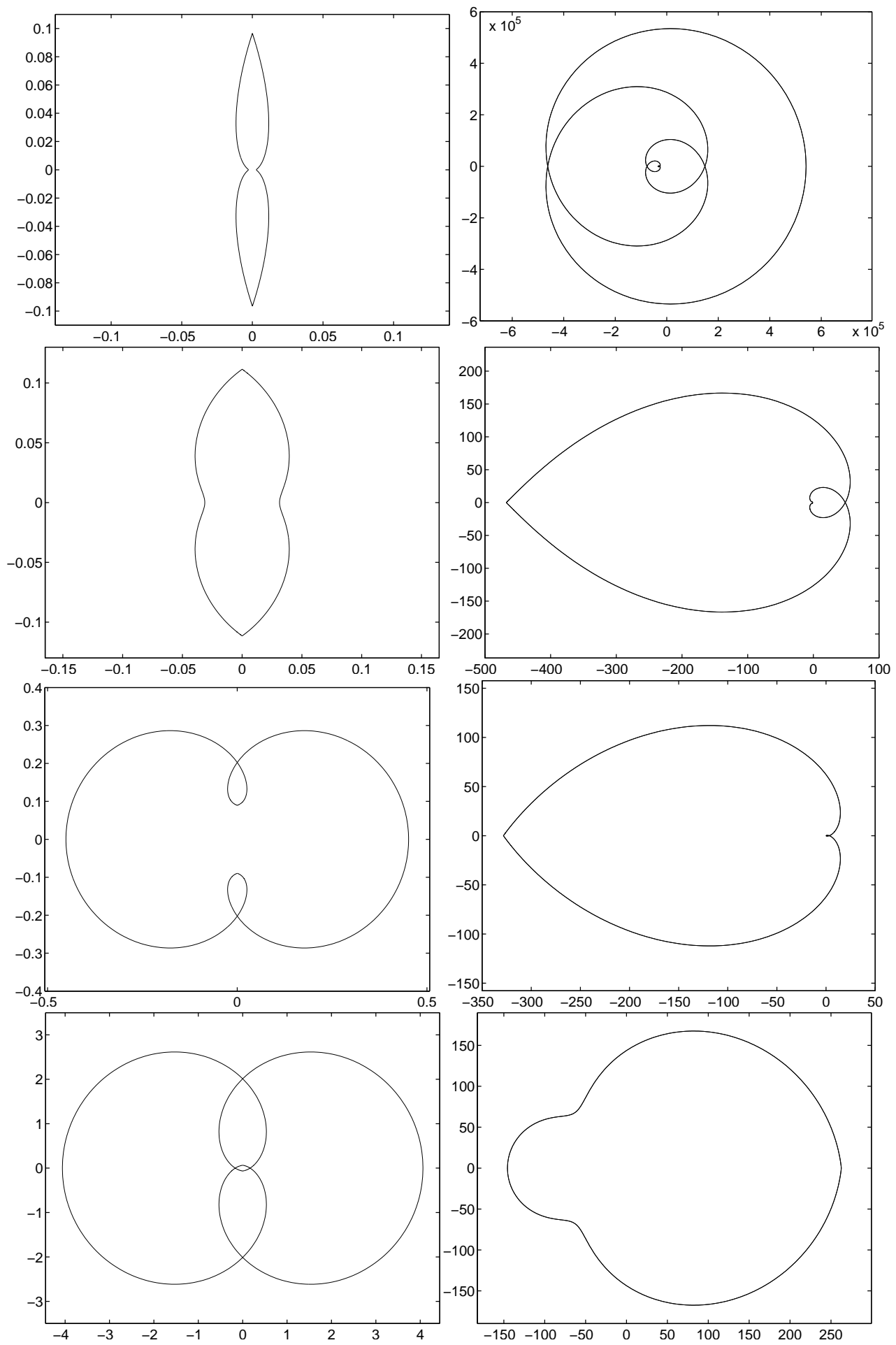

FIG. 3.3. Solution $\phi$ for contour (3.15)-(3.16) with various opening angles $\theta$. Left column: $k=1$, $f(z)=-\bar{z}|z|$ and $\theta=0.2 \pi, 0.6 \pi, 1.4 \pi, 1.8 \pi$. Right column: $k=-1.5, f(z)=-1 / \bar{z}^{10}$ and $\theta=$ $0.2 \pi, 0.5 \pi, 0.6 \pi, 1.8 \pi$. 


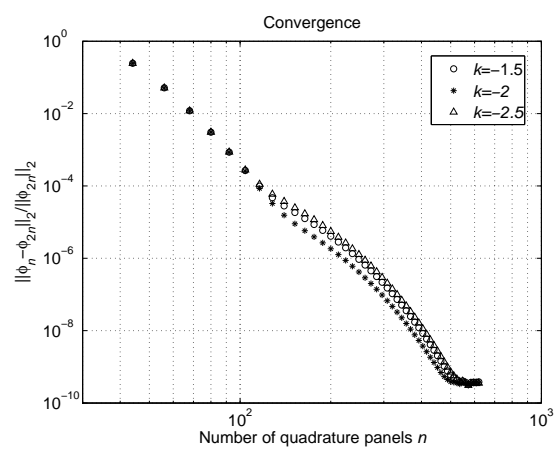

FIG. 3.4. Convergence of $\phi_{n}$ for the contour (3.15)-(3.16) with the opening angle $\theta=0.2 \pi$ and $f(z)=-1 / \bar{z}^{10}$ for $k=-1.5, k=-2$ and $k=-2.5$. The relative error is measured as $\| \phi_{n}-$ $\phi_{2 n}\left\|_{2} /\right\| \phi_{2 n} \|_{2}$.

right-hand side $f$ has no strong oscillation, then there is a less complicated connection between the shape of $\phi$ and the magnitude of $\theta$.

Figure 3.4 confirms an excellent convergence of the method also in the case of a small $\theta$ and a rapidly varying $f$.

4. Fredholm properties and the invertibility of the operators $A_{\tau}$. The previous theorem underscores the importance on the operators $A_{\tau}$ associated with the corner points $\tau \in \mathcal{M}_{\Gamma}$. Thus it is desirable to find effectively verifiable conditions of their invertibility. Unfortunately, at present there is no result related to this problem. The operators under consideration belong to an algebra of Toeplitz operators with piecewise continuous matrix symbols, and there are no efficient methods to check their invertibility. Nevertheless, the Fredholm properties of such operators can be studied. As far as the invertibility is concerned, it seems that only a numerical approach can give us any ideas when such operators are invertible.

Let us start with an auxiliary result.

Lemma 4.1. Let $\tau_{j} \in \mathcal{M}_{\Gamma}$. The operator $A_{\tau_{j}}$ is invertible (Fredholm) if and only if the operator

$$
\begin{aligned}
& \widehat{A}_{\tau_{j}}= \\
& \left(\begin{array}{cccc}
0 & e^{i 2 \beta_{j}} B^{\delta, \varepsilon}\left(\boldsymbol{m}_{2 \pi-\theta_{j}}\right) & -k I & k B^{\delta, \varepsilon}\left(\boldsymbol{n}_{\theta_{j}}\right) \\
-e^{i 2\left(\beta_{j}+\theta_{j}\right)} B^{1-\delta, 1-\varepsilon}\left(\boldsymbol{m}_{\theta_{j}}\right) & 0 & k B^{1-\delta, 1-\varepsilon}\left(\boldsymbol{n}_{\theta_{j}}\right) & -k I \\
-k I & k B^{\delta, \varepsilon}\left(\boldsymbol{n}_{\theta_{j}}\right) & 0 & e^{-i 2 \beta_{j}} B^{\delta, \varepsilon}\left(\boldsymbol{m}_{\theta_{j}}\right) \\
k B^{1-\delta, 1-\varepsilon}\left(\boldsymbol{n}_{\theta_{j}}\right) & -k I & -e^{-i 2\left(\beta_{j}+\theta_{j}\right)} B^{1-\delta, 1-\varepsilon}\left(\boldsymbol{m}_{2 \pi-\theta_{j}}\right) & 0
\end{array}\right)
\end{aligned}
$$

is invertible (Fredholm).

The proof of this result immediately follows from [8, Lemma 1.4.6] and relations (23) of [7].

Let $a$ be an essentially bounded function on the unit circle $\mathbb{T}$, and let $\left(a_{k}\right)$ be the sequence of its the Fourier coefficients. The Toeplitz operator $T(a)$ is defined on finitely supported sequences $\left(\xi_{k}\right)$ by

$$
T(a)\left(\xi_{k}\right)=\left(\eta_{j}\right),
$$

where $\eta_{j}:=\sum_{k=0}^{\infty} a_{j-k} \xi_{k}, j=0,1, \ldots, \infty$. It can be extended on the whole space $l^{2}$ by continuity. 
Consider the smallest closed $C^{*}$-subalgebra $\mathfrak{T}_{2}$ of the algebra of bounded linear operators $\mathfrak{B}\left(l_{2}\right)$ containing all Toeplitz operators $T(a)$ with generating functions $a \in$ $L^{\infty}(\Gamma)$.

LEMMA 4.2. Let $\boldsymbol{k}$ refer to the one of the functions defined by (2.3). Then for any corner point $\tau_{j}$ the entries of the operator $B^{\delta, \varepsilon}(\boldsymbol{k})$ belong to the algebra $\mathfrak{T}_{2}$ and the symbol $\mathcal{A}_{A_{r, p}^{\delta_{r}, \varepsilon_{p}(k)}}$ of the operator $A_{r, p}^{\delta_{r}, \varepsilon_{p}}(\boldsymbol{k})$ is

$$
\mathcal{A}_{A_{r, p}^{\delta_{r}, \varepsilon_{p}(k)}}(z)=\boldsymbol{k}(z), \quad z \in L
$$

Proof. The proof of this result can be obtained following considerations of $[8$, Section 5.4].

Consider now the matrix

$$
\mathcal{A}_{B^{\delta, \varepsilon}(\mathbf{k})}(z):=\left(w_{p} \mathcal{A}_{A_{r p}^{\delta_{r}, \varepsilon_{p}}(\mathbf{k})}(z)\right)_{r, p=0}^{d-1} .
$$

It follows from (4.2) that

$$
\mathcal{A}_{B^{\delta, \varepsilon}(\mathbf{k})}(z)=(\mathbf{W} \otimes \mathbf{k})(z), \quad z \in L,
$$

where $\mathbf{W}:=\left(w_{p}\right)_{r, p=0}^{d-1}$ and $\mathbf{W} \otimes \mathbf{k}$ is the tensor product of $\mathbf{W}$ and $\mathbf{k}$.

Lemma 4.1, Lemma 4.2 and the representations (4.1) and (4.2) imply the following result.

THEOREM 4.3.

1. The operator $A_{\tau_{j}}$ is Fredholm if and only if the determinant $\operatorname{det} \mathcal{A}_{A_{\tau_{j}}}(z)$ of the matrix

$$
\begin{gathered}
\mathcal{A}_{A_{\tau_{j}}}(z)= \\
\left(\begin{array}{cccc}
0 & e^{i 2 \beta_{j}} \boldsymbol{W} \otimes \boldsymbol{m}_{2 \pi-\theta_{j}} & -k I & k \boldsymbol{W} \otimes \boldsymbol{n}_{\theta_{j}} \\
-e^{i 2\left(\beta_{j}+\theta_{j}\right)} \boldsymbol{W} \otimes \boldsymbol{m}_{\theta_{j}} & 0 & k \boldsymbol{W} \otimes \boldsymbol{n}_{\theta_{j}} & -k I \\
-k I & k \boldsymbol{W} \otimes \boldsymbol{n}_{\theta_{j}} & 0 & -e^{-i 2 \beta_{j}} \boldsymbol{W} \otimes \boldsymbol{m}_{\theta_{j}} \\
k \boldsymbol{W} \otimes \boldsymbol{n}_{\theta_{j}} & -k I & e^{-i 2\left(\beta_{j}+\theta_{j}\right)} \boldsymbol{W} \otimes \boldsymbol{m}_{2 \pi-\theta_{j}} & 0
\end{array}\right)(z)
\end{gathered}
$$

does not vanish on the line $z=x+i / 2, x \in \mathbb{R}$.

2. The operator $A_{\tau_{j}}$ is invertible if and only if:

(a) The winding number of the function $\operatorname{det} \mathcal{A}_{A_{\tau_{j}}}(z), z=x+i / 2, x \in \mathbb{R}$ is equal to zero.

(b) The dimension of the kernel $\operatorname{dim} \operatorname{ker} A_{\tau_{j}}=0$.

Note that the condition $2(a)$ can be easily verified. On the other hand, there is no analytic tool to find the dimension of the kernel space of the operator $A_{\tau_{j}}$. However, this condition does not depend on a specific shape on the initial contour but only on the opening angle $\theta_{j} \in(0,2 \pi)$. Therefore, one can try to use special contours and numerical methods to find those values $\theta$ for which $A_{\tau_{j}}$ are not invertible. In particular, in order to decide whether $A_{\tau_{j}}$ is invertible or not, one can run the numerical method for equations on contours with one corner or on contours with multiple corners, all with the same opening angle $\theta_{j}$. In such a specific situation the first part of Theorem 3.4 can be reformulated as follows.

COROLlaRY 4.4. Let $\Gamma$ be a simple closed piecewise smooth contour all corner points $\tau_{j} \in \mathcal{M}_{\Gamma}$ of which have the same opening angle $\theta_{1}$. Then the operator $A_{\tau_{1}}$ is invertible if and only if the sequence $\left(Q_{n}^{\delta} B_{\Gamma, k}^{(\varepsilon, n)} P_{n}^{d}\right)$ is stable. 

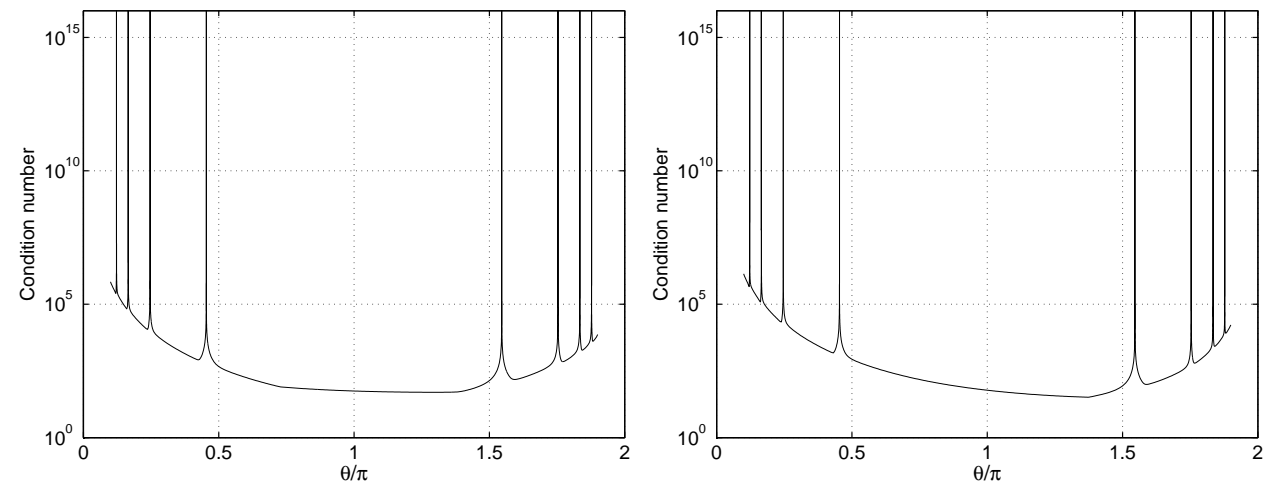

FIG. 4.1. Condition numbers of the discretized operator $B_{\Gamma, 1}$ for different angles $\theta$. Left: the one-corner contour with the original Nyström method. Right: the two-corner contour with the original Nyström method.

Taking this result into account, let us consider the stability of the Nyström method for the Muskhelishvili equation for the two contours of Figure 3.2. On both contours we consider the Nyström method as described in Section 3.1. Computing the corresponding condition numbers for various values of the parameter $\theta$ and representing the results graphically, see Figure 4.1, we conclude there are certain values of $\theta$ where the corresponding operator $A_{\tau}$ is not invertible. These values are represented by the sharp peaks in Figure 4.1.

In these examples, $k=1$ and the two-corner contour is discretized using 100 quadrature panels containing 1600 points, i. e. $n=100, d=16$, and the one-corner contour is discretized using 80 quadrature panels containing 1280 points, i.e. $n=80$, $d=16$.

The peaks for the two-corner contour with the original Nyström method are located at the following $\theta / \pi$ values (three significant digits are given)

$$
0.122, \quad 0.166, \quad 0.246, \quad 0.454, \quad 1.546, \quad 1.754, \quad 1.834, \quad 1.878
$$

The peaks for the one-corner contour with the original Nyström method are located at the following $\theta / \pi$ values (three significant digits are given)

$$
0.122, \quad 0.166, \quad 0.246, \quad 0.454, \quad 1.546, \quad 1.754, \quad 1.834, \quad 1.878
$$

In other words, Figure 4.1 supports the fact that the invertibility of the associated operators $A_{\tau}$ depends on the opening angle but not on the shape of the contour. It is remarkable that the above instability angles coincide up to three digits with the corresponding instability angles of the Nyström method for the Sherman-Lauricella equation [3]. It leads to a conjecture that if $k=1$, then the Nyström method for the Muskhelishvili equation is stable if and only if so is for the Sherman-Lauricella equation. However, the authors were not able to find a rigorous analytic proof for this statement.

On the other hand, critical angles strongly depend on the parameter $k$. Thus for $k=-1.5$ there are only six such angles $\theta \pi$ with $\theta$ taking the values

$$
0.127, \quad 0.196, \quad 0.391, \quad 1.609, \quad 1.804, \quad 1.873,
$$



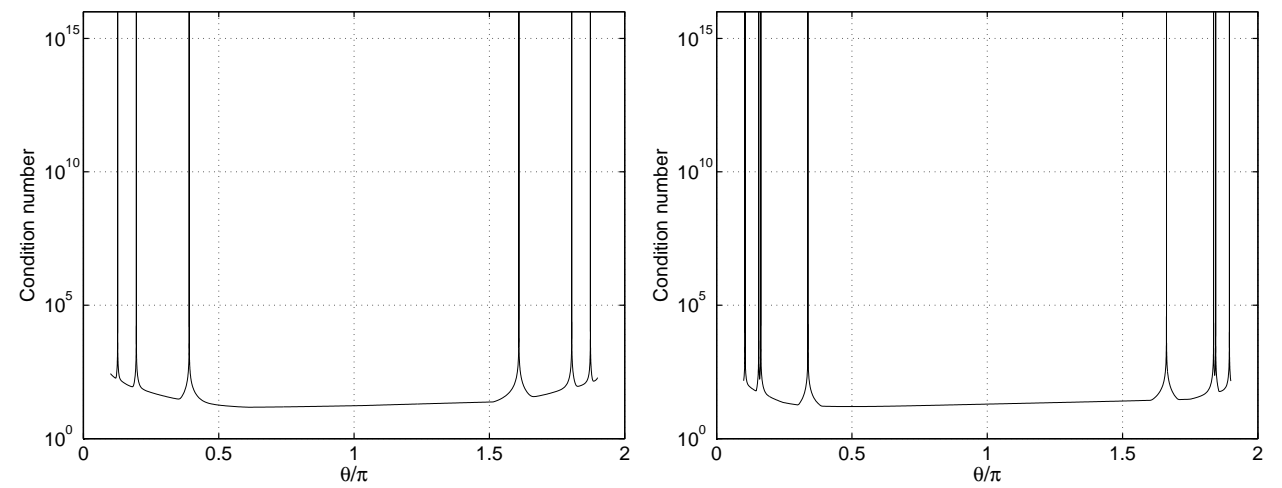

FIG. 4.2. Condition numbers of the discretized operator $B_{\Gamma, k}$ for different angles $\theta$ in the case of one-corner geometry. Left: $k=-1.5$. Right: $k=-2.5$.

and for $k=-2.5$, the corresponding critical values of $\theta$ are

$$
0.105, \quad 0.156, \quad 0.163, \quad 0.338, \quad 1.662, \quad 1.837, \quad 1.844, \quad 1.895
$$

cf. Figure 4.2 .

In conclusion we want to note that while the focus of the present paper is on stability for the original Nyström method, improved computational economy in the solution of integral equations on piecewise smooth contours can be obtained with a recently developed scheme [15]. That scheme uses a modified (product integration) Nyström method [14, Section 2.1] and also employs a compression technique to restrict integral operators to low-dimensional subspaces - thereby reducing the number of discretization points needed to reach a given accuracy. A remarkable property of the modified Nyström method is that it seems to possess no "singular" opening angles. The condition numbers for this method are presented in the right part of Figure 4.3.
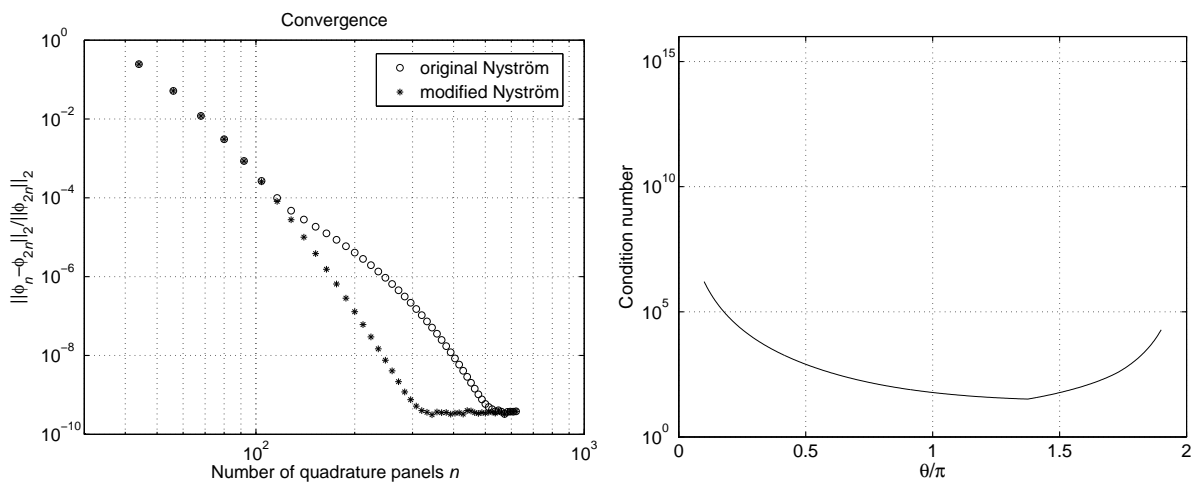

FIG. 4.3. Left: Convergence of the original and modified Nyström methods for the contour (3.15)(3.16) with the opening angle $\theta=0.2 \pi, k=-1.5$ and $f(z)=-1 / \bar{z}^{10}$. Right: Condition numbers of the discretized operator $B_{\Gamma, k}$ for different angles $\theta$ in the two-corner contour and with the modified Nyström method [14, Section 2.1]. 


\section{REFERENCES}

[1] R. H. Chan, T. K. Delillo, And M. A. Horn, The numerical solution of the biharmonic equation by conformal mapping, SIAM J. Sci. Comput., 18 (1997), pp. 15711582.

[2] G. A. Chandler And I. H. SloAn, Spline qualocation methods for boundary integral equations, Numer. Math., 58 (1990), pp. 537-567.

[3] V. D. Didenko And J. Helsing, Features of the Nyström method for the ShermanLauricella equation on piecewise smooth contours, East Asian J. Appl. Math., 1 (2011), pp. $403-414$.

[4] V. D. Didenko And J. Helsing, Stability of the Nyström method for the ShermanLauricella equation, SIAM J. Numer. Anal, 49 (2011), pp. 1127-1148.

[5] V. D. Didenko, S. Roch, And B. Silbermann, Approximation methods for singular integral equations with conjugation on curves with corners, SIAM J. Numer. Anal., 32 (1995), pp. 1910-1939.

[6] V. D. Didenko And B. Silbermann, Extension of $C^{*}$-algebras and Moore-Penrose stability of sequences of additive operators, Linear Algebra Appl., 275/276 (1998), pp. 121140.

[7] V. D. Didenko And B. Silbermann, On stability of approximation methods for the Muskhelishvili equation, J. Comput. Appl. Math., 146 (2002), pp. 419-441.

[8] V. D. Didenko AND B. Silbermann, Approximation of additive convolution-like operators, Frontiers in Mathematics, Birkhäuser Verlag, Basel, 2008. Real $C^{*}$-algebra approach.

[9] V. D. Didenko And E. Venturino, Approximate solutions of some Mellin equations with conjugation, Integral Equations Operator Theory, 25 (1996), pp. 163-181.

[10] V. D. Didenko And E. Venturino, Approximation methods for the Muskhelishvili equation on smooth curves, Math. Comp., 76 (2007), pp. 1317-1339.

[11] R. V. DUDUChAVA, General singular integral equations and fundamental problems of the plane theory of elasticity, Trudy Tbiliss. Mat. Inst. Razmadze Akad. Nauk Gruzin. SSR, 82 (1986), pp. 45-89.

[12] I. C. Gohberg AND I. A. FEL'DMAn, Convolution equations and projection methods for their solution, American Mathematical Society, Providence, R.I., 1974. Translated from the Russian by F. M. Goldware, Translations of Mathematical Monographs, Vol. 41.

[13] J. Helsing, On the interior stress problem for elastic bodies, ASME J. Appl. Mech., 67 (2000), pp. 658-662.

[14] J. HELSING, Integral equation methods for elliptic problems with boundary conditions of mixed type, Journal of Computational Physics, 228 (2009), pp. 8892-8907.

[15] J. HELSING, A fast and stable solver for singular integral equations on piecewise smooth curves, SIAM Journal on Scientific Computing, 33 (2011), pp. 153-174.

[16] C. T. KELleY, A fast multilevel algorithm for integral equations, SIAM J. Numer. Anal., 32 (1995), pp. 501-513.

[17] S. G. Mikhlin AND S. PRÖSSDORF, Singular integral operators, Springer-Verlag, Berlin, 1986.

[18] N. I. Muskhelishvili, A new general method of solution of the fundamental boundary problems of the plane theory of elasticity, Dokl. Akad. Nauk SSSR, 3 (1934), pp. 7-11.

[19] N. I. MuskheliShVILI, Singulyarnye integralnye uravneniya, Izdat. "Nauka", Moscow, augmented ed., 1968. Granichnye zadachi teorii funktsii i nekotorye ikh prilozheniya $\mathrm{k}$ matematicheskoi fizike. [Boundary value problems in the theory of function and some applications of them to mathematical physics], With an appendix by B. Bojarski.

[20] N. I. MuskhelishViLI, Some basic problems of the mathematical theory of elasticity, Noordhoff International Publishing, Leiden, english ed., 1977. Fundamental equations, plane theory of elasticity, torsion and bending, Translated from the fourth, corrected and augmented Russian edition by J. R. M. Radok.

[21] V. Z. PARTON AND P. I. PERLIN, Integral equations in elasticity, "Mir", Moscow, 1982. Translated from the Russian, With a foreword by D. I. Sherman.

[22] S. PRÖSSDORF AND B. SilbermanN, Numerical analysis for integral and related operator equations, Birkhäuser Verlag, Berlin-Basel, 1991.

[23] L. L. SchumaKeR, Spline functions: basic theory, Krieger publishing, Malabar, Florida, 1993. 